\title{
Polysaccharide Vaccine
}

National Cancer Institute

\section{Source}

National Cancer Institute. Polysaccharide Vaccine. NCI Thesaurus. Code C97125.

A category of vaccines that use the extracted and purified outer polysaccharide coat of the particular bacteria. 